\title{
The Apoptotic and Inflammatory Response of Brain Striatal Tissue to Extracorporeal Membrane Oxygenation (ECMO) Following the Model of Cardiac Arrest in Young Piglets
}

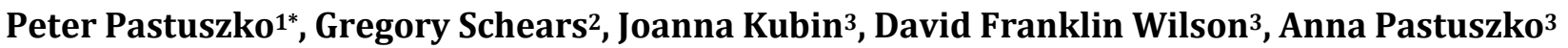 \\ ${ }^{1}$ Mount Sinai Hospital, New York, USA \\ ${ }^{2}$ Mayo Clinic, Department of Anesthesiology, Rochester, USA \\ ${ }^{3}$ Department of Biochemistry \& Biophysics, School of Medicine, University of Pennsylvania, Philadelphia, USA \\ Email: *peter.pastuszko@mountsinai.org
}

How to cite this paper: Pastuszko, P., Schears, G., Kubin, J., Wilson, D.F. and Pastuszko, A. (2021) The Apoptotic and Inflammatory Response of Brain Striatal Tissue to Extracorporeal Membrane Oxygenation (ECMO) Following the Model of Cardiac Arrest in Young Piglets. World Journal of Cardiovascular Diseases, 11, 498-508.

https://doi.org/10.4236/wjcd.2021.1111047

Received: September 21, 2021

Accepted: November 13, 2021

Published: November 16, 2021

Copyright $\odot 2021$ by author(s) and Scientific Research Publishing Inc. This work is licensed under the Creative Commons Attribution International License (CC BY 4.0).

http://creativecommons.org/licenses/by/4.0/ (c) (i) Open Access

\begin{abstract}
We investigated the effects of ECMO on pro-apoptotic and pro-inflammatory signaling in the striatum of piglets following cardiac arrest. 3-week-old piglets were anesthetized, paralyzed and ventilated. Oxygen in the ventilated gas was decreased from $21 \%$ to $7 \%-10 \%$ over $30 \mathrm{~min}$, then ventilation stopped until cardiac arrest. Three minutes after arrest, resuscitation began in two groups, without ECMO (CA) and with ECMO (ECMO). In a control group (C), the animals were sham operated. After 6 hours of recovery, the piglets were euthanized and stratum harvested. Measurement of apoptotic and inflammatory proteins was performed by RayBiotech, Inc. The results are means (6) \pm SEM. There were no differences between CA and ECMO groups for anti-apoptotic proteins. ECMO significantly decreased pro-apoptotic proteins (Bax, cytoC, IGFBP-6, TNF-beta and TRAIR 1 and 3) as compared to CA group. Bcl-2 to Bax ratio increased in ECMO group suggesting that ECMO can least partially protect striatum from apoptotic injury. With respect to inflammation, ECMO significantly decreased both anti-inflammatory (ANG-1, FGF-21, IFN-alpha and beta, IGF-2, IL-10, IL-13, IL-1ra, IL-22, IL-4, IL-6, NCAM-1, SCF, TGF-alpha, TIMP-1and 2, VECF) and pro-inflammatory proteins (IL-12p40, IL-21, IL-15, IL-1 alpha and beta, IL-8, MIP-1 beta, OPG, PIGF-2, RANTES and TGF beta) in striatum of piglets. Conclusions: In a piglet model of cardiac arrest, ECMO significantly reduced levels of pro-apoptotic proteins without changing the levels of anti-apoptotic proteins. ECMO also significantly decreased the levels of both pro- and anti-inflammatory proteins. This decrease in the levels of both pro- and anti-inflammatory proteins may lead to disturbed neuronal metabolism and amplify inflammatory cell death.
\end{abstract}




\section{Keywords}

Brain, ECMO, Apoptosis, Inflammation

\section{Introduction}

Extracorporeal membrane oxygenation (ECMO) is a cardiopulmonary life-saving support system used in critically ill neonates and young children with severe cardiorespiratory failure and congenital heart disease [1]. ECMO has reduced the mortality by about $80 \%$, but in most cases it causes multi-system organ dysfunction [2] [3].

The mechanisms of the adverse consequences of ECMO are still not fully understood. It is, however, established that ECMO induces the systemic inflammatory response syndrome (SIRS) characterized by a "cytokine storm" that may importantly contribute to various post-ECMO injuries. Inflammation involves a disruption of the delicate balance between pro-inflammatory and anti-inflammatory mediators; an unbalance in this system can lead to injuries of multiple organs. Both clinical studies and animals' experiments indicated that ECMO changes this balance by increasing levels of pro-inflammatory cytokines in serum and tissue [4] [5] [6] [7]. The increased serum concentrations of inflammatory cytokines can be caused by their increased de novo synthesis or/and their redistribution from tissue into plasma [5].

In the brain, an increase of pro-inflammatory cytokines can trigger apoptosis and necrosis [8] [9], impair the blood-brain barrier and increase its permeability [10], cause injury of glial and neuronal cells [11], increase free radicals [12]. These biochemical changes may facilitate embolic strokes, hypoxic-ischemic encephalopathy, cerebral infarction, intracranial and subarachnoid hemorrhages, seizures and cerebral edema [13]. The inflammatory response is detectable within the first few hours of ECMO.

In this study, we used a porcine model of cardiac arrest to investigated effect of ECMO following cardiac arrest on key pro- and anti-inflammatory proteins in the striatum, the main input site within the basal ganglia. We also investigated anti- and pro-apoptotic proteins and changes in their ratio because the ratio was a sensitive index of susceptibility to apoptotic injury. Apoptosis, particularly in neonates and young children, plays a major role in neuronal cell death after hypoxia-ischemia, brain trauma, and neurodegenerative diseases [14].

\section{Abbreviations and Acronyms}

ECMO (Extracorporeal membrane oxygenation), Bcl-2 (B-cell lymphoma 2), Bcl-w (B-cell lymphoma-w), HSP (Heat shock proteins 27, 60 and 70), IGFBP (insulin-like growth factor binding protein 1and 3), cytoC (cytochrome C), Bax (Bcl-2-associated X protein), IGFBP-6 (insulin-like growth factor binding protein 6), TNF-beta (Tumor Necrosis Factor-beta), TRAILR 1 and 3 (Tumor ne- 
crosis factor related apoptosis-inducing ligand 1 and 3), ANG-1(Angiogenin 1), FGF-21(Fibroblast growth factor -21), IFN-alpha and beta (Interferon alpha and beta), IGF-2 (insulin-like growth factor 2), IL-4, 6, 8, 10, 13,15, 21, 22, IL-12p40, IL-1alpha, IL-1beta (Proteins of Interleukins Family), IL-1ra (interleukin-1 receptor antagonist), NCAM-1 (Neural cell adhesion molecule 1), SCF (Skp, Cullin, F-box containing complex-a multi-protein E3 ubiquitin ligase complex), TGF alpha and beta (Transforming growth factor alpha and beta), TIMP 1 and 2 (a tissue inhibitor of metalloproteinases), VEGF (Vascular endothelial growth factor), MIP-1 beta(Macrophage inflammatory protein), OPG (Osteoprotegerin), PIGF-2 (Placental growth factor), RANTES (an acronym for Regulated upon Activation, Normally T-Expressed, and presumably Secreted).

\section{Material and Methods}

Animal Model: Eighteen male piglets from different litters, approximately 3week-old and weighing 4 - $5 \mathrm{~kg}$, were obtained from Meck Swine, LLC (Lancaster, PA) and were used within 4 days of delivery. The piglets were anesthetized with $4 \%$ isoflurane, intubated and mechanically ventilated with air/30\% oxygen $\left(\mathrm{O}_{2}\right)$ mixture with ventilation parameters appropriate to maintain normocapnia. Anesthesia was maintained with $1.5 \%$ - 3\% isoflurane supplemented with IV infusion of morphine $(0.1-0.3 \mathrm{mg} / \mathrm{kg} / \mathrm{h})$. A femoral vein and arterial catheters were placed. Blood gases $\left(\mathrm{PaCO}_{2}, \mathrm{PaO}_{2}\right), \mathrm{pH}$, hemoglobin, electrolytes and glucose concentrations were monitored using i-STAT blood gas machine (Abbot Point of Care Inc., Princeton, NJ). Mean arterial blood pressure (MAP), ECG and rectal temperature were monitored throughout the study.

All animal procedures were carried out in strict accordance with the NIH Guide for the Care and Use of Laboratory Animals and were approved by the Institutional Animal Care and Use Committee of the University of Pennsylvania, Philadelphia, PA (protocol number 805971).

Experimental groups: the animals were randomly assigned to one of three groups: 1) Circulatory arrest (CA group, $\mathrm{n}=6$ ), 2) CA with ECMO (ECMO group, $n=6$ ), and 3) Control (sham-operated, $n=6$ ). The animals in the control group were anesthetized, intubated and mechanically ventilated for $6 \mathrm{hrs}$ in the same manner as the animals in the other two groups.

Experimental protocol: after stabilization, inspiratory $\mathrm{pO}_{2}$ was gradually decreased from $21 \%$ to $7 \%$ - $10 \%$ over 30 min after which ventilation was stopped until the occurrence of heart arrest. Three min before the end of hypoxic period a short acting neuromuscular blocker, Vecuronium $(0.2-1.0 \mathrm{mg} / \mathrm{kg})$ was administered IV. Three min after heart arrest, resuscitation was begun with restoration of mechanical ventilation and chest compressions. If needed, epinephrine (0.02 $0.1 \mathrm{mg} / \mathrm{kg}$ IV) was injected. The usual code dose is $0.1 \mathrm{mg} / \mathrm{kg}$ but frequently smaller dose is giving because it overshoots on the blood pressure. The pediatric defibrillator was used to restart the heart rhythm.

If the resuscitation was successful: 
- CA group was ventilated for $1 \mathrm{hr}$ with $100 \% \mathrm{O}_{2}$ and then for 5 hrs with $30 \% \mathrm{O}_{2}$;

- ECMO group had the carotid vessels cannulated and was placed on ECMO; $500 \mathrm{U}$ heparin was administered IV and perfusion flow rate was set at approx. $150 \mathrm{ml} / \mathrm{kg} / \mathrm{min}$. Body temperature was maintained at $36^{\circ} \mathrm{C}$ for $6 \mathrm{hrs}$ with arterial blood pressure, blood gases, $\mathrm{pH}$, glucose monitored at $30 \mathrm{~min}$ intervals and adjusted, if needed.

The ECMO circuit consisted of a Cobe Roller Pump (Cobe, Lakewood, CO), a membrane oxygenator with integrated arterial filter (Capiox FX05, Terumo Cardiovascular Systems Corp., Ann Arbor, MI), and Sarns Heater-Cooler System (Terumo). The circuit was primed with Plasmalyte-A (Baxter Healthcare Corp., Deerfield, Ill.) and 25\% albumin. Porcine donor whole blood (Innovative Research Inc., MI) was added to maintain a hematocrit value of approximately $30 \%$. Heparin (1000 units), calcium chloride (150 mg), sodium bicarbonate (25 $\mathrm{mEq}$ ) were added to the pump prime.

At the end of each experiment, while still anesthetized, the piglets were euthanized with an IV injection of Euthasol $(0.2 \mathrm{ml} / \mathrm{kg})$, the brains were removed, dissected and stored at $-80^{\circ} \mathrm{C}$ for further analysis.

Determination of striatal protein levels: total protein was extracted from about $100 \mathrm{mg}$ of striatal tissue and the levels of apoptotic and inflammatory proteins were determined by RayBiotech, Inc. (Norcross, GA).

Statistical analysis: data were analyzed using one-way ANOVA looking for statistically significant differences in the three possible configurations: 1) $\mathrm{C}$ vs. CA group; 2) C vs. ECMO group; and 3) CA vs. ECMO group. Protein levels are presented as percentage of the mean in the $C$ (sham operated) group.

\section{Results}

\section{Effect of hypoxia and ECMO on physiological parameters in piglets}

Systolic blood pressure, mean blood pressure, Heart rate, $\mathrm{pH}, \mathrm{pCO}_{2}$, the percent saturation of oxygen in the blood $\left(\mathrm{SPO}_{2}\right)$ were measured before hypoxia, at the end of hypoxia, at the end of CPR (rhythm of hear return) and after 1, 3 and 5 hrs of recovery. The significant difference between hypoxia and ECMO group was observed only in $\mathrm{SPO}_{2}$ at the end of hypoxia. $\mathrm{SPO}_{2}$ decreased from $98.5 \pm$ 0.84 to $16.75 \pm 5.4$ in hypoxic group and $48.6 \pm 9.8$ in ECMO group ( $<<0.05$ ). There were not significant differences in SPO2 between hypoxia and ECMO groups in other measured time points.

Effect of ECMO on immunoreactivities of striatal anti- and pro-apoptotic proteins

Immunoreactivities of the proteins involved in apoptotic and inflammatory responses were determined in the striatum of piglet brains following cardiac arrest and 6 hrs of post-cardiac arrest recovery with or without ECMO.

Effect of ECMO on anti- and pro-apoptotic proteins is presented on Figure 1. The striatal levels of anti-apoptotic proteins-Bcl-2, Bcl-w, HSP27, HSP60, HSP70, IGFBP-1, IGFBP-3, Livin, Survivin, didn't change significantly between 
experimental groups. Concurrently, ECMO significantly decreased the levels of six pro-apoptotic proteins as compared to CA group (Bax from 99\% to $72 \%(\mathrm{p}<0.05)$; cytochrome $\mathrm{C}$ from $115 \%$ to $71 \%$ ( $\mathrm{p}<0.05)$; IGFBP-6 from $115 \%$ to $76 \%(\mathrm{p}<0.05)$; transforming growth factor $\beta$ (TGF $\beta$ ) from $113 \%$ to $74 \%$ ( $\mathrm{p}<0.05$ ); TRAIR-1 from $106 \%$ to $57 \%(\mathrm{p}<0.05)$; TRAIR-3 from $89 \%$ to $48 \%(\mathrm{p}<0.05))$. The calculated Bcl-2 to Bax ratio in ECMO group as compared to CA group increased by $30 \%$.

\section{Effect of ECMO on striatal anti-inflammatory proteins}

The levels of 17 anti-inflammatory proteins that were significantly lower in ECMO group as compared to CA group are presented on Figure 2.

The proteins that have only anti-inflammatory responses are: ANG-1 decreased from $189 \%$ to $106 \%$ ( $<<0.025$ ); FGF-21 from $102 \%$ to $31 \%(\mathrm{p}<0.01$ ); IFN- $\alpha$ from $65 \%$ to $28 \%$ (p < $<.05$ ); IFN- $\beta$ from $138 \%$ to $57 \%$ (p < 0.01$)$ : IGF-2 from $137 \%$ to $47 \%$ ( $<<0.01$ ); IL-10 from $120 \%$ to $63 \%$ (p < 0.05 ); IL-13 from 95 to $12 \%(\mathrm{p}<0.005)$; IL-1 receptor antagonist (IL-1ra) from $134 \%$ to $56 \%(\mathrm{p}<$
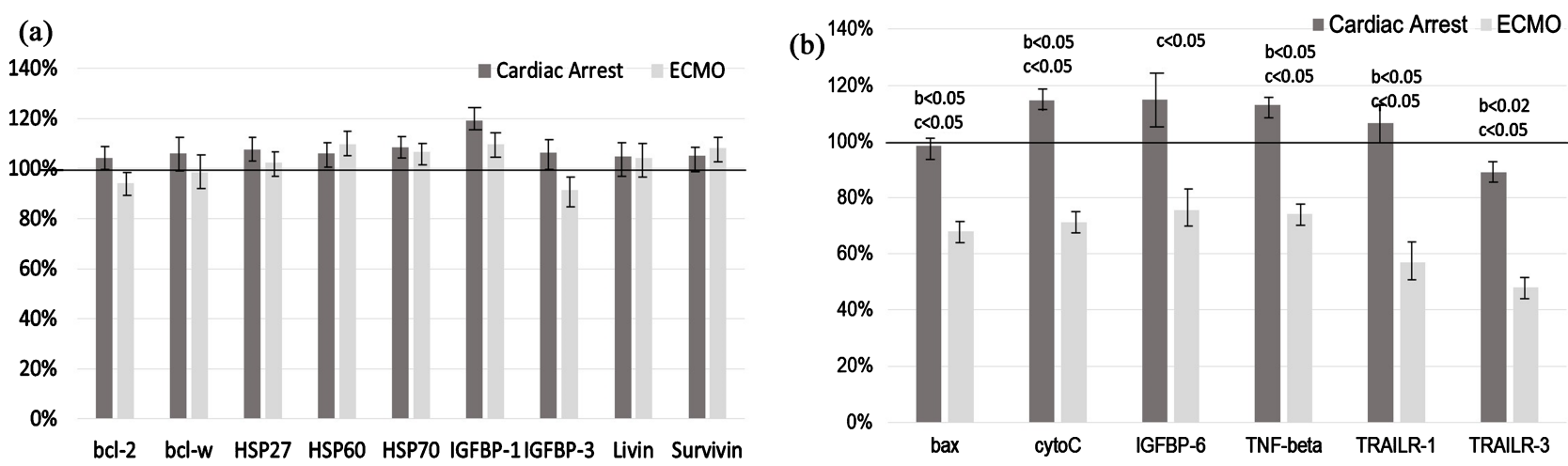

Figure 1. Effect of ECMO on levels of anti-apoptotic (A) (Figure 1(a)) and pro-apoptotic proteins (Figure 1(b)) in striatum of piglets. Protein levels are presented as percentage of the mean in the $\mathrm{C}$ (sham operated) group. Bars represent the means \pm SEM for the density of the bands for six independent experiments. $\mathrm{p}<0.05$ was considered statistically significant difference between the indicated groups. Data were analyzed using one-way ANOVA looking for statistically significant differences in the three possible configurations: (a) C vs. CA group; (b) C vs. ECMO group; and (c) CA vs. ECMO group.
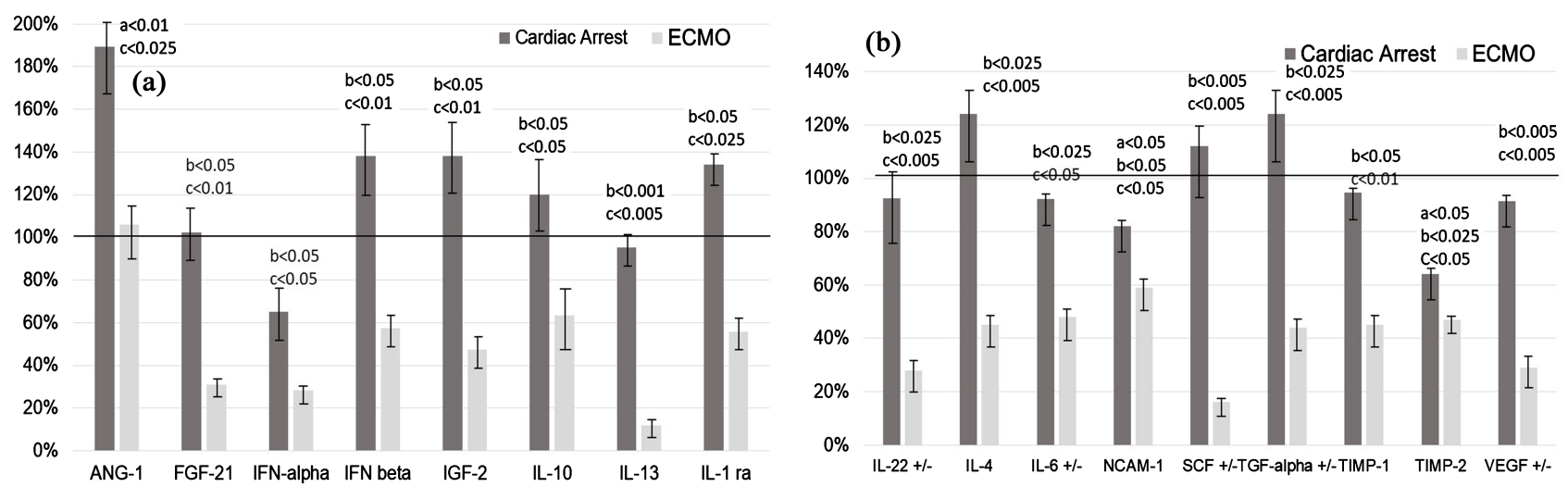

Figure 2. Effect of ECMO on levels of anti-inflammatory in striatum of piglets. Protein levels are presented as percentage of the mean in the $\mathrm{C}$ (sham operated) group. Bars represent the means \pm SEM for the density of the bands for six independent experiments. $\mathrm{p}<0.05$ was considered statistically significant difference between the indicated groups. Data were analyzed using one-way ANOVA looking for statistically significant differences in the three possible configurations: (a) C vs. CA group; (b) C vs. ECMO group; and (c) CA vs. ECMO group. 
0.025); IL-4 from $124 \%$ to $45 \%$ ( $\mathrm{p}<0.005$ ); NCAM-1 from $83 \%$ to $58 \%$ ( $\mathrm{p}<$ 0.05 ) and TIMP- 1 from $95 \%$ to $45 \%(\mathrm{p}<0.01)$ and TIMP-2 from $68 \%$ to $47 \%$ (p $<0.05)$. They are presented on Figure 2(a).

The five proteins presented in Figure 2(b) also significantly decreased in ECMO group as compared to CA group but, depending on other concurrent changes, they can exert either anti-inflammatory or pro-inflammatory effects. They were: IL-6 decreased from $92 \%$ to $48 \%$ ( $\mathrm{p}<0.05$ ); IL-22 from $92 \%$ to $28 \%(\mathrm{p}<0.005)$; SCF from $112 \%$ to $16 \%(\mathrm{p}<0.005)$; TGF $\alpha$ from $124 \%$ to $44 \%(\mathrm{p}<0.005)$; and VEGF from $91 \%$ to $29 \%$ ( $\mathrm{p}<0.005)$.

\section{Effect of ECMO on striatal pro-inflammatory proteins}

Effect of ECMO on 11 pro-inflammatory proteins is shown on Figure 3.

As compared to CA group, ECMO group has significantly decreased levels of IL-12p40 from $94 \%$ to $42 \%$ ( $<<0.05)$; IL-21 from $179 \%$ to $55 \%(\mathrm{p}<0.005)$; IL-15 from $114 \%$ to $16 \%$ ( $<<0.025)$; IL- $1 \alpha$ from $87 \%$ to $51 \%(\mathrm{p}<0.05)$, IL- $1 \beta$ from $44 \%$ to $5 \%$ ( $\mathrm{p}<0.005$ ), IL-8 from 89 to $44 \%$ ( $\mathrm{p}<0.05$ ); MIP- $1 \beta$ from $104 \%$ to $72 \%$ ( $\mathrm{p}<0.05$ ), OPG from $94 \%$ to $31 \%(\mathrm{p}<0.05)$, PIGF-2 from $134 \%$ to $96 \%$ $(\mathrm{p}<0.05)$, RANTES from $103 \%$ to $44 \%(\mathrm{p}<0.005)$ and TGF $\beta$ from $93 \%$ to $52 \%$ $(\mathrm{p}<0.05)$.

\section{Discussion}

The purpose of our study was to determine the effects of ECMO applied following cardiac arrest on the levels of apoptotic and inflammatory proteins in the striatum of piglets. Striatum is involved in the control of motor behavior and various aspects of behavioral plasticity and is highly sensitive to hypoxia/ischemia-induced injury among different brain regions.

\section{Effect of ECMO on anti- and pro-apoptotic proteins}

We found no significant differences between CA and ECMO groups for anti-apoptotic proteins. In contrast, there were significant differences between the two groups in the levels of pro-apoptotic proteins. Six pro-apoptotic proteins
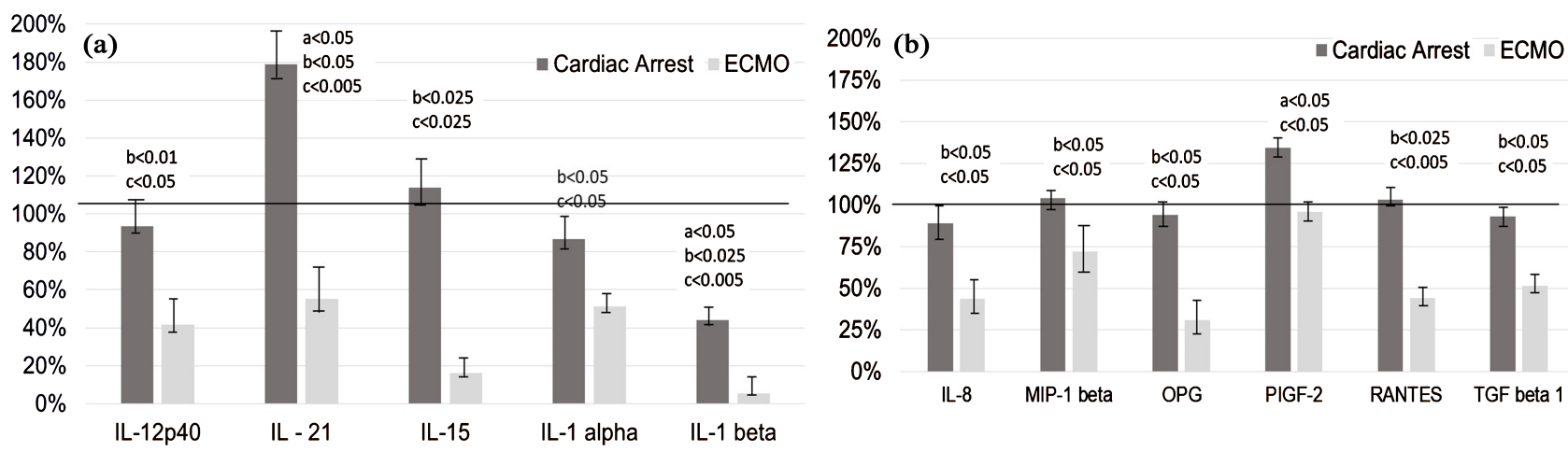

Figure 3. Effect of ECMO on levels of pro-inflammatory in striatum of piglets. Protein levels are presented as percentage of the mean in the $\mathrm{C}$ (sham operated) group. Bars represent the means \pm SEM for the density of the bands for six independent experiments. $\mathrm{p}<0.05$ was considered statistically significant difference between the indicated groups. Data were analyzed using one-way ANOVA looking for statistically significant differences in the three possible configurations: (a) C vs. CA group; (b) C vs. ECMO group; and (c) CA vs. ECMO group. 
were significantly decreased in CA with ECMO group as compared to CA without ECMO group. Of particular importance among the proteins that control apoptosis are those that belong to the Bcl-2 family, with Bcl-2 and Bax being most significant. Bcl-2 has anti-apoptotic effects and enhances cell survival possibly by regulating cytosolic and intranuclear $\mathrm{Ca}^{2+}$ concentrations [15]. In contrast, the pro-apoptotic protein Bax promotes cell death by activating caspases [16]. The active form of Bcl-2 heterodimerizes with Bax and their ratio determines the cellular susceptibility to apoptotic stimuli [17] [18]. Accordingly, an increased ratio of Bax to $\mathrm{Bcl}-2$ increases predisposition to apoptosis in hypoxic and hypocapnic newborn brains.

Based on our findings, ECMO does not change Bcl-2 level but significantly decreases Bax level by about 30\%. Therefore, the Bcl-2 to Bax ratio increased, suggesting that ECMO offers protection from apoptotic injury.

\section{Effect of ECMO on inflammatory response in the striatum of piglets}

Inflammatory proteins are regulators of multiple responses to infection, immune responses, inflammation, and trauma. Cytokines include chemokines, interferons, interleukins, lymphokines and tumor necrosis factor. Some cytokines increase inflammation (pro-inflammatory), whereas others reduce inflammation and promote healing (anti-inflammatory). Major anti-inflammatory cytokines include IL-1ra, IL-4, IL-10, IL-11, and IL-13. The major pro-inflammatory cytokines responsible for early inflammatory responses are IL- $1 \alpha$, IL- $1 \beta$ and TNF $\alpha$. IL-6 and transforming growth factor (TGF $\beta$ ) and, depending on the conditions, can have either anti-inflammatory or pro-inflammatory actions. The inflammatory responses are determined by the balance between pro- and anti-inflammatory cytokines. We found that ECMO significantly decreased the levels of major anti-inflammatory proteins, including IL-4, IL-6, IL-10, IL-13, IL-1ra, as well as major pro-inflammatory proteins (IL- $1 \alpha, \operatorname{IL}-1 \beta$, TGF $\beta$ ). The level of $\operatorname{TNF} \alpha$, a major pro-inflammatory protein, did not differ between the CA and ECMO groups.

The decrease in pro-inflammatory proteins found in our study in ECMO group is in agreement with the recent study of Zhang et al. [19], who reported that in pig brain cortex ECMO applied after cardiac arrest decreased the levels of pro-inflammatory cytokines: IL-1, IL-6, TNF $\alpha$ and TGF $\beta$.

The pro-inflammatory proteins included in our study play key roles in inflammatory responses. Both animal experiments and clinical studies suggest that increased circulating pro-inflammatory cytokines, such as IL- $1 \beta$, IL-6, and TNF $\alpha$, are associated with increased mortality [20]. Notably, inhibition of IL- $1 \beta$ converting enzyme or deletion of IL- $1 \beta$ and IL- $1 \alpha$ results in markedly reduced ischemic damage and neuronal death.

$\operatorname{TGF} \beta$ is another key inflammatory protein. Some studies suggest that this cytokinase can have a protective effect [21] but it mainly functions as a pro-inflammatory protein that can cause damage of the blood-brain barrier, hemorrhage, neuroinflammation, cell death [22] and increase expression of IL-1 $\beta$ and TNF $\alpha$ [23]. 
IL-6 is a powerful pro-inflammatory cytokine that is essential for the inflammatory acute phase response induced by tissue damage. An increased plasma concentration of IL- 6 levels in children after cardiopulmonary bypass were associated with poorer neuromotor performance and, therefore, possible damage in the striatum [24]. However, this protein can also act as anti-inflammatory cytokinase by inhibiting TNF and IL-1 production.

Collectively our study indicates that ECMO, by decreasing the level of important pro-inflammatory proteins can have protective effect in the striatum. However, we also found a decrease in several key ant-inflammatory proteins whose major function is to inhibit the synthesis of pro-inflammatory cytokines. In particular, IL-10 is the most important anti-inflammatory cytokine found within the human immune response. It prevents immunopathology in several diseases and disease models, both in the central nervous system and peripheral organs [25] [26]. Another key anti-inflammatory protein that decreased in ECMO group is IL-1ra (IL-1receptor antagonist). IL-1ra functions as a specific inhibitor of the two other functional members of the IL- 1 family, IL- $1 \alpha$ and IL- $1 \beta$. IL-1ra blocks the action of IL- $1 \alpha$ and IL- $1 \beta$ by competitive inhibition at the IL- 1 receptor level. IL-1ra has been reported to decrease hypoxic-ischemic injury [27] [28] and reduced the brain injury and death of the cranial nerves. Consistent with these findings, neonatal mice deficient in IL-1 converting enzyme are resistant to hypoxic-ischemic insults, and inhibition of endogenous IL-1 activity with IL-1ra protects against ischemic injury [29]. Similarly, inhibition of IL-1b converting enzyme or deletion of IL- $1 \alpha$ and IL- $1 \beta$ results in markedly reduced ischemic damage and neuronal death.

Because ECMO decreases both ant-inflammatory and pro-inflammatory proteins it is not clear whether ECMO changes the balance between the two antagonistic protein groups and, therefore, has a net protective or damaging effect on striatal tissue. If we look at the changes in IL- $1 \alpha$ and IL- $1 \beta$ levels and their inhibitor IL-1ra, we can see that IL- $1 \alpha$ and IL- $1 \beta$ decreased by $36 \%-39 \%$ and IL-ra decreased by $78 \%$. This would suggest that ECMO has more prominent effect on IL- 1 ra than on IL- $1 \alpha$ and IL- $1 \beta$. From this can be postulate that ECMO will have negative effect on striatal tissue by disproportionately stronger effect on the anti-apoptotic IL-1ra than on the pro-apoptotic IL- $1 \alpha$ and IL- $1 \beta$.

What mechanisms lead to the changes in anti- and pro-inflammatory proteins uncovered in our study is unclear and will require future investigation. The study of Mcilwain [5] on neonatal porcine model of ECMO has shown that TNF $\alpha$ and IL- 8 concentrations rose faster in plasma than in the peripheral tissues during ECMO. They concluded that the rising plasma levels of these cytokines immediately following the initiation of ECMO could not be completely explained on the basis of increased de novo synthesis in inflamed tissues and may have resulted, at least partially, from a redistribution of preformed stores of these cytokines. One possible explanation for the decrease of inflammatory proteins in striatal tissue observed in our study would be that they are release from 
tissue to plasma, thereby depleting the corresponding tissue levels; this will be required future investigation.

\section{Limitations of Our Study}

The limitation of our study is that we determine apoptotic and inflammatory protein levels at one time point, at $6 \mathrm{hrs}$ following CA. There could be earlier transient changes and changes that occurred at longer times after recovery and were not captured in our study. Indeed, there are reports that different pro-inflammatory proteins are up- or down-regulated at different time points following a variety of hypoxic-ischemic insults. For example, IL6 has a short half-life in plasma and peaks at $2 \mathrm{hrs}$ after onset of cardiopulmonary bypass. Hedtjarn et al. [30] measured expression of 148 genes related to the immune-inflammatory response in immature brain following hypoxia-ischemia (HI). They reported that at $2 \mathrm{hrs}$ after $\mathrm{HI}$ only three genes were upregulated, but at $8 \mathrm{hrs}$ the number of significantly upregulated genes had increased to 49 . Twenty-four and $72 \mathrm{hrs}$ after HI, 77 and as many as 119 genes were, respectively, upregulated. We selected $6 \mathrm{hrs}$ as our target time point because it fitted well with a typical duration of clinical surgical procedures with ECMO. Still, to better elucidate the interactions among the pro- and anti-apoptotic and pro- and anti-inflammatory proteins in the context of fundamental mechanisms promoting and impeding preservation of brain function following ECMO, it would be of interest to study both shorter and longer time intervals than the 6 hrs period used in our study. It would also be of interest concurrently measure tissue, plasma and cerebrospinal fluid levels of the key proteins of interest. Our present findings should help in the design of future studies.

\section{Acknowledgements}

The study was supported by SEED fund from Mount Sinai School of Medicine, New York.

\section{Conflicts of Interest}

The authors declare no conflicts of interest regarding the publication of this paper.

\section{References}

[1] Wang, C., Zhang, L., Qin, T., Xi, Z., Sun, L., Wu, H. and Li, D. (2020) Extracorporeal Membrane Oxygenation in Trauma Patients: A Systematic Review. World Journal of Emergency Surgery, 15, 51-65. https://doi.org/10.1186/s13017-020-00331-2

[2] Robinson, S. and Peek, G. (2015) The Role of ECMO in Neonatal \& Pediatric Patients. Pediatric Child Health, 25, 222-227. https://doi.org/10.1016/j.paed.2015.03.005

[3] Madderom, M.J., Reuser, J.J., Utens, E.M., et al. (2013) Neurodevelopmental, Educational and Behavioral Outcome at 8 Years after Neonatal ECMO: A Nationwide Multicenter Study. Intensive Care Medicine, 39, 1584-1593. 
https://doi.org/10.1007/s00134-013-2973-1

[4] Ortega, S.B., Pandiyan, P., Windsor, J., Torres, V.O., Selvaraj, U.M., Lee, A., Morriss, M., Tian, F., Raman, L. and Stowe, A.M. (2019) A Pilot Study Identifying Brain-Targeting Adaptive Immunity in Pediatric Extracorporeal Membrane Oxygenation Patients with Acquired Brain Injury. Critical Care Medicine, 47, 206-213. https://doi.org/10.1097/CCM.0000000000003621

[5] MclLwain, R.B., Timpa, J.G., Kurundkar, A.R., Holt, D.W., Kelly, D.R., Hartman, Y., et al. (2010) Plasma Concentrations of Inflammatory Cytokines Rise Rapidly during ECMO-Related SIRS Due to the Release of Preformed Stores in the Intestine. Laboratory Investigation, 90, 128-139. https://doi.org/10.1038/labinvest.2009.119

[6] Chen, Q., Yu, W., Shi, J., Shen, J., Hu, Y., Gao, T., Zhang, J., Xi, F., Gong, J., Li, J. and Li, N. (2013) The Effect of Venovenous Extra-Corporeal Membrane Oxygenation (ECMO) Therapy on Immune Inflammatory Response of Cerebral Tissues in Porcine Model. Journal of Cardiothoracic Surgery, 8, 186-194.

https://doi.org/10.1186/1749-8090-8-186

[7] Karagiannidi, C., Brodie, D., Strassmann, S., Stoelben, E., Philipp, A., Bein, T., et al. (2016) Extracorporeal Membrane Oxygenation: Evolving Epidemiology and Mortality. Intensive Care Medicine, 42, 889-896.

https://doi.org/10.1007/s00134-016-4273-z

[8] Stolp, H.B. and Dziegielewska, K.M. (2009) Review: Role of Developmental Inflammation and Blood-Brain Barrier Dysfunction in Neurodevelopmental and Neurodegenerative Diseases. Neuropathology and Applied Neurobiology, 35, 132-146. https://doi.org/10.1111/j.1365-2990.2008.01005.x

[9] Aktas, O., Ullrich, O., Infante-Duarte, C., Nitsch, R. and Zipp, F. (2007) Neuronal Damage in Brain Inflammation. Archives of Neurology, 64, 185-189.

https://doi.org/10.1001/archneur.64.2.185

[10] Webb, A.A. and Muir, G.D. (2000) The Blood-Brain Barrier and Its Role in Inflammation. Journal of Veterinary Internal Medicine, 14, 399-411. https://doi.org/10.1111/j.1939-1676.2000.tb02248.x

[11] Nimmo, A.J., Cernak, I., Heath, D.L., Hu, X., Bennett, C.J. and Vink, R. (2004) Neurogenic Inflammation Is Associated with Development of Edema and Functional Deficits Following Traumatic Brain Injury in Rats. Neuropeptides, 38, 40-47. https://doi.org/10.1016/j.npep.2003.12.003

[12] Yang, M.S., Min, K.J. and Joe, E. (2007) Multiple Mechanisms That Prevent Excessive Brain Inflammation. Journal of Neuroscience Research, 85, 2298-2305. https://doi.org/10.1002/jnr.21254

[13] Kim, H., Hysi, P.G., Pawlikowska, L., Poon, A., Burchard, E.G., Zaroff, J.G., Sidney, S., Ko, N.U., Achrol, A.S., Lawton, M.T., McCulloch, C.E., Kwok, P.-Y. and Young, W.L. (2009) Common Variants in Interleukin-1-Beta Gene Are Associated with Intracranial Hemorrhage and Susceptibility to Brain Arteriovenous Malformation. Cerebrovascular Diseases, 27, 176-182. https://doi.org/10.1159/000185609

[14] Lo, A.C., Houenou, L.J. and Oppenheim, R.W. (1995) Apoptosis in the Nervous System: Morphological Features, Methods, Pathology, and Prevention. Archives of Histology and Cytology, 58, 139-149. https://doi.org/10.1679/aohc.58.139

[15] Marin, M.C., Fernandez, A., Bick, R.J., Brisbay, S., Buja, L.M., Snuggs, M., McConkey, D.J., von Eschenbach, A.C., Keating, M.J. and McDonnell, A.T.J. (1996) Apoptosis Suppression by Bcl-2 Is Correlated with Regulation of Nuclear and Cytosolic $\mathrm{Ca}^{2+}$. Oncogene, 12, 2259-2266.

[16] Golstein, P. (1997) Controlling Cell Death. Science, 275, 1081-1082. 
https://doi.org/10.1126/science.275.5303.1081

[17] Gillardon, F., Wickert, H. and Zimmermann, M. (1995) Up-Regulation of Bax and Down Regulation of Bcl-2 Is Associated with Kainite-Induced Apoptosis in Mouse Brain. Neuroscience Letters, 192, 85-88. https://doi.org/10.1016/0304-3940(95)11619-8

[18] Oltvai, Z.N., Milliman, C.L. and Korsmeyer, S.J. (1993) Bcl-2 Heterodimerizes in Vivo with a Conserved Homolog, Bax, That Accelerates Programmed Cell Death. Cell, 74, 609-619. https://doi.org/10.1016/0092-8674(93)90509-O

[19] Zhang, Y., Li, C.-S., Yuan, X.-L., Ling, J.-Y., Zhang, Q., Liang, Y., Liu, B. and Zhao, L.-X. (2019) ECMO Attenuates Inflammation Response and Increases ATPase Activity in Brain of Swine Model with Cardiac Arrest Compared with CCPR. Bioscience Reports, 39, BSR20182463. https://doi.org/10.1042/BSR20182463

[20] Niemann, J.T., Rosborough, J.P., Youngquist, S., Shah, A.P., Lewis, R.J., Phan, Q.T., et al. (2009) Cardiac Function and the Proinflammatory Cytokine Response after Recovery from Cardiac Arrest in Swine. Journal of Interferon \& Cytokine Research, 29, 749-758. https://doi.org/10.1089/jir.2009.0035

[21] Vogel, T., Ahrens, S., Buttner, N. and Krieglstein, K. (2010) Transforming Growth Factor Beta Promotes Neuronal Cell Fate of Mouse Cortical and Hippocampal Progenitors in Vitro and in Vivo: Identification of Nedd9 as an Essential Signaling Component. Cerebral Cortex, 20, 661-671. https://doi.org/10.1093/cercor/bhp134

[22] Manaenko, A., Lekic, T., Barnhart, M., Hartman, R. and Zhang, J.H. (2014) Inhibition of Transforming Growth Factor-Beta Attenuates Brain Injury and Neurological Deficits in a Rat Model of Germinal Matrix Hemorrhage. Stroke, 45, 828-834. https://doi.org/10.1161/STROKEAHA.113.003754

[23] Saghazadeh, A., Ferrari, C.C. and Rezaei, N. (2016) Deciphering Variability in the Role of Interleukin-1 Beta in Parkinson's Disease. Reviews in the Neurosciences, 27, 635-650. https://doi.org/10.1515/revneuro-2015-0059

[24] Gessler, P., Schmitt, B., Prêtre, R. and Latal, B. (2009) Inflammatory Response and Neurodevelopmental Outcome after Open-Heart Surgery in Children. Pediatric Cardiology, 30, 301-305. https://doi.org/10.1007/s00246-008-9354-5

[25] Gabay, C. (2006) Interleukin-6 and Chronic Inflammation. Arthritis Research \& Therapy, 8, S3. https://doi.org/10.1186/ar1917

[26] Londono, D., Carvajal, J., Strle, K., Kim, K.S. and Cadavid, D. (2011) IL-10 Prevents Apoptosis of Brain Endothelium during Bacteremia. The Journal of Immunology, 186, 7176-7186. https://doi.org/10.4049/jimmunol.1100060

[27] Hagberg, H., Gilland, E., Bona, E., et al. (1996) Enhanced Expression of Interleukin (IL)-1 and IL-6 mRNA and Bioactive Protein after Hypoxia-Ischemia in Neonatal Rats. Pediatric Research, 40, 603-609. https://doi.org/10.1203/00006450-199610000-00015

[28] Martin, D., Chinookoswong, N. and Miller, G. (1994) The Interleukin-1 Receptor Antagonist (rhIL-1ra) Protects against Cerebral Infarction in a Rat Model of Hypoxia-Ischemia. Experimental Neurology, 130, 362-367. https://doi.org/10.1006/exnr.1994.1215

[29] Relton, J.K. and Rothwell, N.J. (1992) Interleukin-1 Receptor Antagonist Inhibits Ischaemic and Excitotoxic Neuronal Damage in the Rat. Brain Research Bulletin, 29, 243-246. https://doi.org/10.1016/0361-9230(92)90033-T

[30] Hedtjarn, M., Mallard, C. and Hagberg, H. (2004) Inflammatory Gene Profiling in the Developing Mouse Brain after Hypoxia-Ischemia. Journal of Cerebral Blood Flow \& Metabolism, 24, 1333-1351. https://doi.org/10.1097/00004647-200412000-00003 